\title{
Distinguishing Lipedematous Scalp, Lipedematous Alopecia, and Diffuse Alopecia Areata
}

\author{
Rachita S. Dhurat Sanober Burzin Daruwalla Smita Sunil Ghate \\ Mithali Mahendra Jage Aseem Sharma \\ Department of Dermatology, LTMMC and LTMGH, Sion Hospital, Mumbai, India
}

\section{Established Facts}

- Soft, spongy, or doughy consistency of the scalp resulting from thickening of the subcutaneous layer is termed as lipedematous scalp.

- When such soft, boggy scalp is associated with hair loss, where the scalp hair is no longer than a few centimeters, it is referred as lipedematous alopecia.

\section{Novel Insights}

- Alopecia areata, a treatable condition, in the setting of boggy consistency of the scalp can be misdiagnosed as lipedematous alopecia.

- Other causes of alopecia should always be ruled out in order to pick up conditions which are essentially reversible and treatable, and thereby help decrease patient morbidity.

- LA and LS are two different manifestations of the same disease, i.e. lipedematous scalp.

\section{Keywords}

Lipedematous scalp · Alopecia areata $\cdot$ CD3 + T-lymphocytes

\begin{abstract}
Soft, spongy or doughy consistency of the scalp resulting from thickening of the subcutaneous layer is termed as lipedematous scalp (LS). When such soft, boggy scalp is associated with hair loss, where the scalp hair is no longer than a few centimeters, it is referred as lipedematous alopecia (LA). There have been coincidental reports of androgenetic alo-
\end{abstract}

pecia, psoriasis, mucinosis, and discoid lupus erythematosus with LS. We report a case of LS and alopecia areata whose characteristic histopathological feature showed a "swarm of bees" appearance and thickening of subcutaneous tissue. The diagnosis of alopecia areata was further confirmed by immunohistochemistry staining of the tissue showing CD3+ T-lymphocytes around the bulb. The aim of this case report is to make readers understand that LA and LS are not different diseases and one needs to rule out other causes of alopecia in order to pick up conditions which are essentially reversible and treatable.

(c) 2019 S. Karger AG, Base

\section{KARGER}

(c) 2019 S. Karger AG, Basel

E-Mail karger@karger.com

www.karger.com/sad
Dr. Rachita Dhurat

Department of Dermatology, LTMMC and LTMGH

Sion Hospital, Dr. Babasaheb Ambedkar Road, Sion West

Mumbai 400022 (India)

E-Mail rachitadhurat@yahoo.co.in 


\section{Introduction}

Lipedematous scalp (LS) is characterized by thickening of the subcutaneous layer of the scalp that results in a soft, spongy, boggy, or doughy consistency of the scalp. When such soft, boggy scalp is associated with hair loss, where the scalp hair is no longer than a few centimeters, it is referred as lipedematous alopecia (LA) [1]. Diffuse pain, paresthesia, or itching and scalp dysesthesia (burning sensation) have been reported in LA and LS patients. Coincidental findings of androgenetic alopecia, psoriasis, mucinosis, and discoid lupus erythematosus with possible associations have been reported. However, LS concomitant with alopecia areata has not been reported to date.

\section{Case Report}

A 52-year-old married woman presented with hair loss for 5 years. She was diagnosed with hypothyroidism for which she was taking levothyroxine for 8 years. She was treated with topical fluocinolone and systemic steroids for hair loss which resulted in regrowth of hair 4 years ago. However, there was recurrence of hair loss in the last year. On examination, there was diffuse hair thinning over the frontal, vertex, and parietal region of the scalp (Fig. 1). Some of her hair was grey. There was no evidence of scalp inflammation, scaling, or increased hair fragility. Hair pull test was negative. The entire scalp was boggy and spongy in consistency and could be pressed easily as if indenting a ping pong ball (Fig. 2). On trichoscopy, exclamation mark hairs, black or yellow dots, and miniaturized hairs were absent. Complete blood count, liver function test, thyroid function test and lipid profile were within normal limits. High-frequency ultrasonography showed an increase in the thickness of subcutaneous tissue, ranging from 6 to $7.2 \mathrm{~mm}$, and a total scalp thickness of $10 \mathrm{~mm}$.

Histopathology of a 4-mm punch biopsy of the scalp was done. It revealed normal epidermis with decrease in the number of follicular units. There was peribulbar lymphocytic infiltrate arranged in a characteristic "swarm of bees" appearance along with melanin in the fibrous stela in the subcutaneous tissue (Fig. 3). Immunohistochemistry staining of the tissue showed CD3+ T-lymphocytes around the bulb further confirming the diagnosis of alopecia areata (Fig. 4). Also noted was increased thickness of subcutaneous fat and absence of connective tissue septa separating subcutaneous tissue into fat lobules leaving a continuous diffuse sheet of mature adipocytes, which is consistent with the finding of LS (Fig. 5). She was started on diphenylcyclopropenone contact immunotherapy (0.01-0.1\%) after sensitization. Hair regrowth was appreciable as early as 2 months of therapy.

\section{Discussion/Conclusion}

In 1935, Cornbleet [2] first described a 44-year-old black woman whose scalp felt as if it were underlaid with soft cotton batting. He reported unusually thick subcutaneous fat layer on histopathology, with no abnormalities of the hair. In 1965, Coskey et al. [1] reported 2 cases with shortened hair and thickening of the scalp. They introduced the term "lipedematous alopecia" and described it as a condition that results in an inability to grow hair longer than $2 \mathrm{~cm}$ in addition to the increased thickness of the subcutaneous fat layer of the scalp. Later, in 1994, Lee et al. [3] reported a case of thickening of the subcutaneous fat layer of the scalp with no hair abnormality and proposed the term "lipedematous scalp" in order to differentiate it from LA. Yasar et al. [4], investigated the clinical and histopathological features of LS and LA in a 7-year retrospective re-evaluation of 31 patients. They reported no statistical difference in the clinical and histopathological findings between these two conditions and proposed that in the pathogenesis of LA, there is an increase in pressure on the hair follicles from the resulting thickening of the subcutaneous fat causing impairment of nutrition of the hair matrix. Thus, this may result in shortening of the hair cycle, decrease in hair growth rate, or both. This
Fig. 1. a Diffuse hair thinning over the frontal area of the scalp. b Diffuse hair thinning over the vertex and parietal region of the scalp with greying of hair.
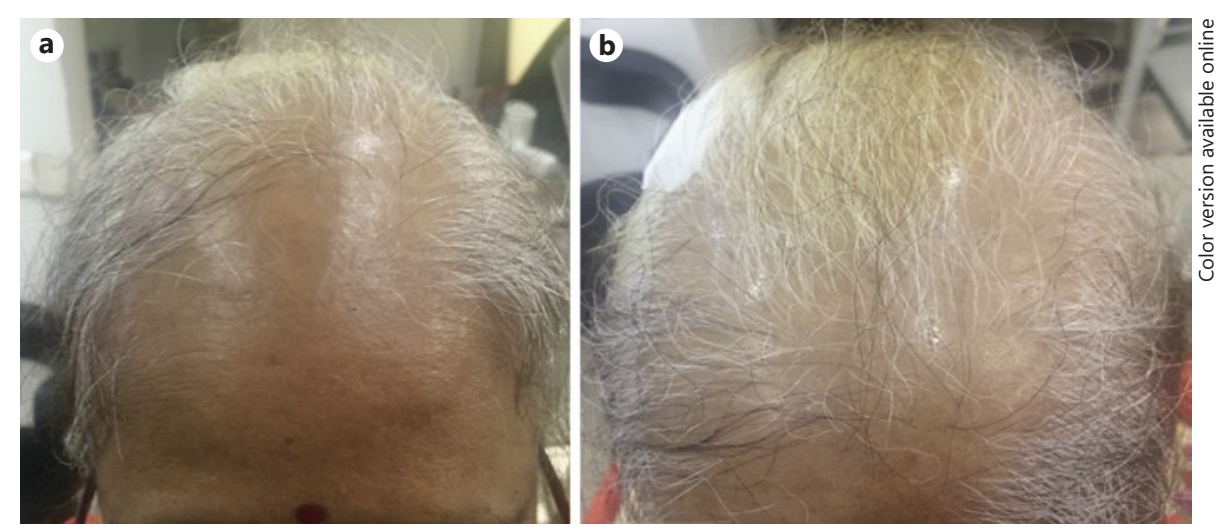

Lipedematous Scalp and Diffuse Alopecia Areata
Skin Appendage Disord 2019;5:316-319 DOI: $10.1159 / 000495947$ 
Fig. 2. Indentation of the scalp due to the boggy, spongy consistency.

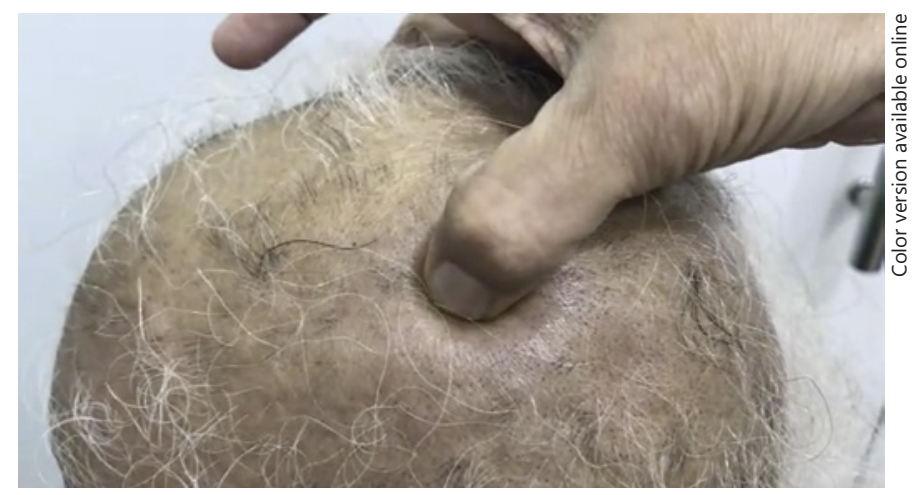

Fig. 3. a Transverse section showing peribulbar lymphocytic infiltrate arranged in a characteristic "swarm of bees" appearance. H\&E. $\times 400$. b Transverse section showing presence of melanin in fibrous stela in the subcutaneous tissue. $\mathrm{H} \& \mathrm{E} . \times 400$.
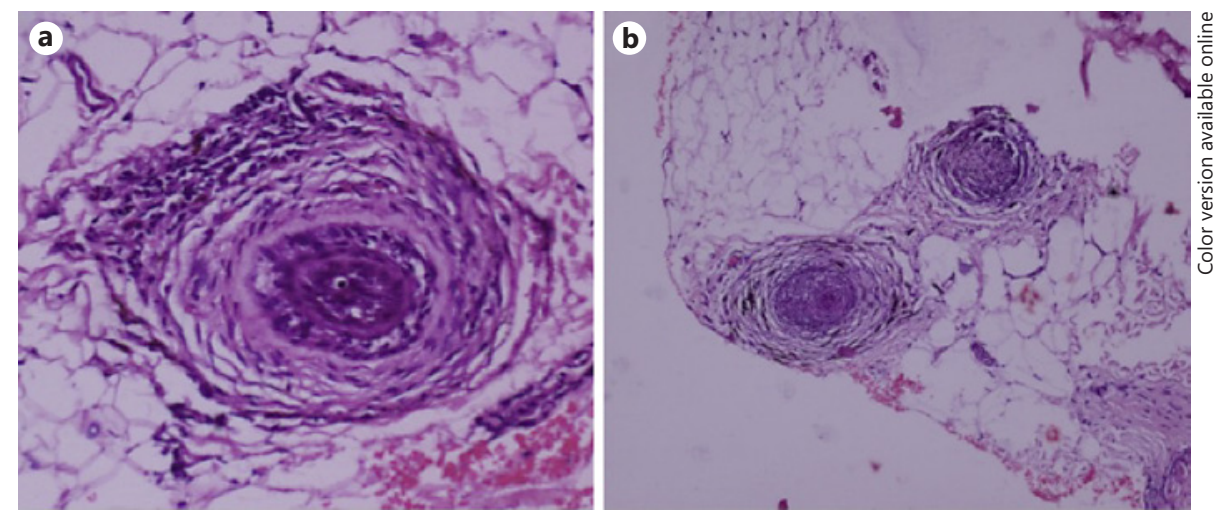

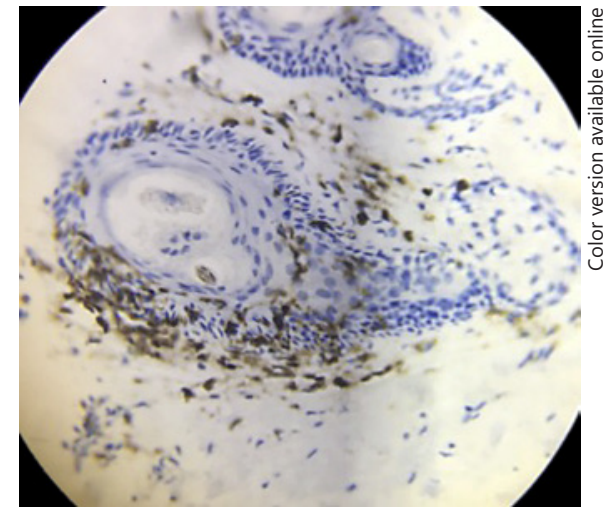

Fig. 4. Presence of CD3+ T-lymphocytes around the bulb. CD3 immunostaining. $\times 400$.

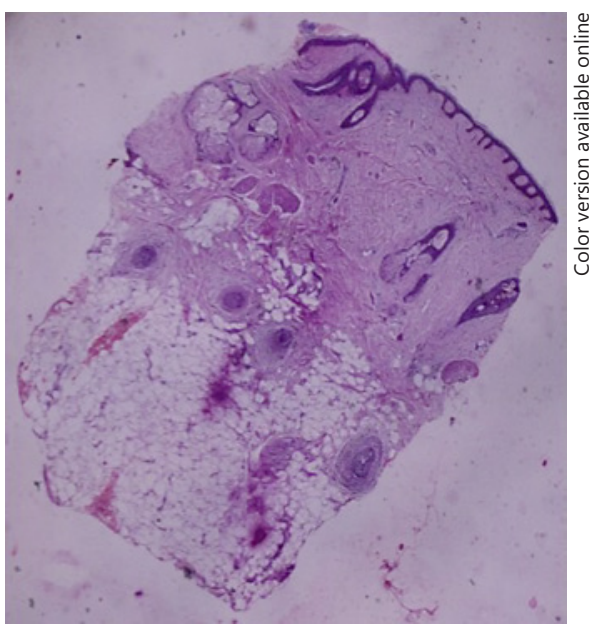

Fig. 5. Increased thickness of subcutaneous fat and absence of connective tissue septa separating subcutaneous tissue into fat lobules leaving a continuous diffuse sheet of mature adipocytes. H\&E. $\times 40$. 
might be responsible for the clinical appearance of scalp hair no longer than a few centimeters and associated hair loss in areas corresponding to the soft, boggy scalp area. Since LA does not develop in all cases of LS, even though thickening occurs, they explained the increased subcutaneous tissue might not be enough to alter the microenvironment of hair follicles in all cases of LS, and there may be interpersonal phenotypical variations in the manifestation of alopecia. Therefore, we agree with Yasar et al. [4] that LA and LS are two different manifestations of the same disease, i.e. LS.

Histopathology of LS and LA classically reveals thickened subcutaneous tissue. In addition, mucin accumulation, dermal edema, lymphatic dilatation, collagen fragmentation, elastic tissue fragmentation, reduced hair follicle count, perifollicular lymphocytic infiltration, perifollicular fibrosis, and dermal lymphocytic infiltration have been reported $[4,5]$. The presence of a peribulbar lymphocytic infiltrate, the so-called "hive of bees," is pathognomonic of alopecia areata [6]. This finding has not been reported in cases of LA as seen in our case. The presence of CD3+ T-lymphocytes within empty follicular tracts or around the bulb on immunohistochemistry reliably favors alopecia areata over patterned hair loss [6]. In our case, CD3+ T-lymphocytes were present around the bulb. With a lifetime incidence of alopecia areata being approximately $2 \%$ worldwide, it is possible that the presence of alopecia areata in our case of LS is purely coincidental and a first of a kind to be reported.

LA has little to no effective treatment to halt or delay progression. Treatment with topical and intralesional steroids and hydroxychloroquine have been tried with no success $[7,8]$. Surgical debulking with scalp reduction has an acceptable result with no evidence of relapse even after 12 months [9]. Cabrera et al. [10] successfully treated a case of LA with mycophenolate mofetil. In our case, alopecia areata, a treatable condition, in the setting of a boggy consistency of the scalp could have been misdiagnosed as LA. Therefore, other causes of alopecia should always be ruled out in order to pick up conditions which are essentially reversible and treatable, and thereby help decrease patient morbidity.

\section{Statement of Ethics}

The authors have no ethical conflicts to disclose.

\section{Disclosure Statement}

The authors have no conflicts of interest to declare.

\section{Author Contributions}

Contributor
$\begin{array}{lllll}1 & 2 & 3 & 4 & 5\end{array}$

\section{Concepts}

Design

Definition of intellectual content

Literature search

Clinical studies

Experimental studies

Data acquisition

Data analysis

Statistical analysis

Manuscript preparation

Manuscript editing

Manuscript review

Guarantor

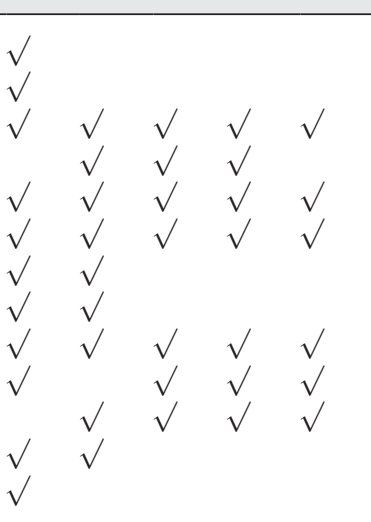

\section{References}

1 Coskey RJ, Fosnaugh RP, Fine G. Lipedematous alopecia. Arch Dermatol. 1961 Oct; 84(4):619-22.

2 Cornbleet T. Cutis verticis gyrata? Lipoma? Arch Dermatol Syphilol. 1935;32:688-98.

3 Lee JH, Sung YH, Yoon JS, Park JK. Lipedematous scalp. Arch Dermatol. 1994 Jun; 130(6):802-3.

4 Yaşar S, Mansur AT, Göktay F, Sungurlu F, Vardar Aker F, Ozkara S. Lipedematous scalp and lipedematous alopecia: report of three cases in white adults. J Dermatol. 2007 Feb; 34(2):124-30.
5 Kane KS, Kwan T, Baden HP, Bigby M. Woman with new-onset boggy scalp. Arch Dermatol. 1998 Apr;134(4):499-500, 502-3.

6 Kolivras A, Thompson C. Distinguishing diffuse alopecia areata (AA) from pattern hair loss (PHL) using CD3(+) T cells. J Am Acad Dermatol. 2016 May;74(5):937-44.

7 Kavak A, Yuceer D, Yildirim U, Baykal C, Sarisoy HT. Lipedematous scalp: a rare entity. J Dermatol. 2008 Feb;35(2):102-5.
8 High WA, Hoang MP. Lipedematous alopecia: an unusual sequela of discoid lupus, or other co-conspirators at work? J Am Acad Dermatol. 2005 Aug;53(2 Suppl 1):S157-61.

9 Yip L, Mason G, Pohl M, Sinclair R. Successful surgical management of lipoedematous alopecia. Australas J Dermatol. 2008 Feb;49(1): 52-4.

10 Cabrera R, Larrondo J, Whittle C, Castro A, Gosch M. Successful treatment of lipedematous alopecia using mycophenolate mofetil. Acta Derm Venereol. 2015 Nov;95(8):10112. 